\title{
BIG BATH ACCOUNTING E TURNOVER DE EXECUTIVOS EM EMPRESAS LISTADAS NA B3
}

\author{
Big Bath Accounting and turnover of executives at companies listed on \\ the B3
}

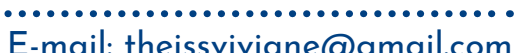

Doutora em Contabilidade pela Universidade Federal de Santa Catarina; Mestre em Ciências Contábeis pela Fundação Universidade Regional de Blumenau; Professora na Universidade do Estado de Santa Catarina. Endereço para contato: Rua Dr. Getúlio Vargas, 2822, 89140-000, Ibirama, Santa Catarina, Brasil. http://orcid.org/0000-0002-2450-9704

Henrique Portulhak

E-mail: henrique.portulhak@ufpr.br

Doutor em Contabilidade pela Universidade Federal do Paraná; Mestre em Contabilidade pela Universidade Federal do Paraná; Professor na Universidade Federal do Paraná. https://orcid.org/0000-0002-9097-2481

Marcos Roberto Kuhl

E-mail: marcosrobertokuhl@yahoo.com.br

Doutor em Administração pela

Universidade Federal do Paraná; Mestre em Ciências Contábeis pela Universidade Federal do Paraná; Professor na Universidade Estadual do Centro-Oeste. https://orcid.org/0000-0001-6578-5804

Romualdo Douglas Colauto

E-mail: rdcolauto.ufpr@gmail.com Doutor em Engenharia de Produção pela Universidade Federal de Santa Catarina; Mestre em Engenharia de Produção pela Universidade Federal de Santa Catarina; Professor na

Universidade Federal do Paraná. https://orcid.org/0000-0003-3589-9389 


\section{Resumo}

O turnover de um executivo pode tornar-se uma justificativa plausível para um maior aproveitamento de acúmulos discricionários em um determinado período, com consequente evidenciação de um suposto prejuízo maior para a empresa, com o intuito de informar ao mercado, em períodos posteriores, aparentes melhoras nos resultados da organização, o que configura o aproveitamento de acúmulos discricionários por meio da modalidade de gerenciamento de resultados denominada Big Bath Accounting (BBA). Nesse contexto, o objetivo do estudo foi identificar em que medida a mudança de executivos em companhias listadas na B3 está associada com o gerenciamento de resultados. Foram realizadas 1.407 observações de 254 empresas, no período de 2009 a 2014, utilizando-se o modelo de Dechow, Hutton, Kim, e Sloan (2012) para a detecção de gerenciamento de resultados, informações e disponibilidades pela base de dados Bloomberg ${ }^{\circledR}$ para a identificação dos eventos de turnover, sendo utilizado o tamanho (logaritmo natural do Ativo) como variável de controle. A amostra apresenta uma concentração de executivos com idade superior a 50 anos, grande rotatividade desse cargo, com preferência pela nomeação de pessoas internas para a função de CEO e maior representatividade de turnover no ano 2013. Os testes realizados não encontraram indícios de que a mudança do executivo provoca $B B A$ em razão da variação de accruals discricionários no período. Da mesma forma, notou-se a existência não confirmada de diferenças entre a contratação de executivos insiders e outsiders para a utilização de mais gastos discricionários. Apesar da falta de comprovação estatística, o estudo ajuda a compreender os conceitos de BBA e incentiva a quebra de paradigmas, por meio de evidências empíricas de turnover de executivos, no que concerne a essa modalidade de gerenciamento de resultados.

Palavras-chave: Gerenciamento de resultados. Big Bath Accounting. Turnover de executivos.

\section{Abstract}

An executive's turnover can become a plausible justification for a greater use of discretionary accumulations in a given period, with consequent evidence of a supposed greater loss to the company, with the intention to informing the market, in later periods, of apparent improvements in the results of the organization, which configures the use of discretionary accumulations through the mode of income management called Big Bath Accounting (BBA). In this context, the objective of the study was to identify the extent to which the change of executives in companies listed on B3 is associated with income management. $A$ total of 1,407 observations were made from 254 companies from 2009 to 2014, using Dechow, Hutton, Kim, and Sloan (2012) model for detection income management, information available by the Bloomberg ${ }^{\circledast}$ database to identify the turnover events, using the size (natural logarithm of the Asset) as control variable. The sample has a concentration of executives over 50 years of age, with a high turnover in this position, with preference for the appointment of internal persons to the role of CEO and greater representation of turnover in the year 2013.The tests carried out didn't find any evidence that BBA changes due to the variation of discretionary accruals in the period. Likewise, there was an unconfirmed existence of differences between the hiring of insiders and outsiders executives for the use of more discretionary spending. Despite the lack of statistical evidence, the study helps to understand the BBA concepts and encourages the breaking of paradigms, through empirical evidence of executive turnover, regarding this mode of results management.

Keywords: Income management. Big Bath Accounting. Executives turnover. 


\section{INTRODUÇÃO}

A rotatividade de funcionários é considerada um dos grandes problemas organizacionais, e, quando se trata da mudança do diretor executivo (CEO) da empresa, esse problema é ainda mais severo por envolver custos transacionais com as mudanças de aspectos estratégicos da companhia, custos trabalhistas rescisórios, treinamentos e adaptações de novos colaboradores para o cargo. Além disso, tem-se a possibilidade de modificações em procedimentos contábeis decorrentes de discricionariedades próprias em casos de substituição de CEOs. A discricionariedade refere-se a escolhas contábeis que podem resultar em provisionamentos de despesas, sobrecarregadas em determinados períodos, a ponto de caracterizar a intencionalidade da empresa em gerenciar resultados, nesse caso, configurando uma modalidade de gerenciamento de resultados denominada Big Bath Accounting (BBA), caracterizada por um tratamento discricionário de itens contábeis com o intuito de reduzir o lucro em um ano, quando já se sabe que a empresa está em um período ruim (Sunder, 2014).

Com o intuito de justificar a redução de lucros e tirar proveito das dificuldades que ocorrem na empresa, a mudança do executivo pode sinalizar aos stakeholders uma preocupação da corporação em tomar atitudes proativas para reverter o atual estágio em que a empresa se encontra (Hazarika, Karpoff, \& Nahata, 2012). Conforme Sunder (2014), - BBA pode ser um fator que explica a instalação de um novo grupo gerencial, como forma de culpar a gestão anterior de eventuais problemas organizacionais transitórios. $O$ mesmo autor expõe que, nos EUA, as companhias provocam o BBA como pretexto para realizar custos de reestruturação, de forma a gerar um saudável crescimento de lucros em anos subsequentes. Nesse contexto, o BBA é frequentemente acompanhado pela troca de gestão e ciclos de suavização, que podem ocorrer várias vezes dentro da mesma organização (Sunder, 2014).

O estudo de Bornemann, Kick, Pfingsten, e Schertler (2015) identificou na rotatividade do cargo de Diretor-executivo de bancos alemães uma justificativa para o aumento das despesas discricionárias no ano de posse do novo CEO. O estudo de Hazarika et al. (2012) também observou situações de gerenciamento de resultados quando a rotatividade do executivo foi forçada, enquanto em situações de mudanças voluntárias ou planejadas não se observaram indícios de gerenciamento de resultados. Dal Magro, Klann, e Mondini (2018) verificaram que nos primeiros anos do mandato de executivos há o incentivo para a declaração de resultados que atendam às expectativas do mercado.

Situações como estas reforçam os problemas de assimetria de informações entre principais e agentes (Jensen \& Meckling, 1976). O novo executivo da empresa pode apropriarse de um evento controverso como este para justificar o reconhecimento de acumulações 
discricionárias e reduzir os resultados da empresa, para, em períodos seguintes, apresentar um comportamento de suavização de lucros, com o intuito de demonstrar uma melhora nos resultados da companhia após a sua permanência na gestão (Sunder, 2014). Esses aspectos unem o interesse de maximizar o valor da empresa e o favorecimento de futuros incentivos aos executivos, todavia, acabam por impactar a qualidade da informação contábil, principalmente no que se refere às suas características qualitativas fundamentais (relevância e representação fidedigna) e de melhorias (comparabilidade, verificabilidade, tempestividade e compreensibilidade), assim como determina a postergação de pagamentos de dividendos direcionados aos acionistas da companhia (Comitê de Pronunciamentos Contábeis [CPC], 2011).

Diante do contexto apresentado, destaca-se a questão de pesquisa: em que medida a mudança de executivos em empresas listadas na B3 está associada com o gerenciamento de resultados na modalidade $B B A$ ? De acordo com o cenário apresentado, o estudo pretende identificar em que medida a mudança de executivos em empresas listadas na B3 está associada com o $B B A$, já que se acredita que haja relação significativa entre BBA em empresas brasileiras e, mais especificamente, na contratação de executivos outsiders. Para sustentar esta hipótese, utilizam-se os estudos de Hazarika et al. (2012), Bornemann et al. (2015) e Sunder (2014).

As contribuições teóricas deste estudo procuram compreender os conceitos de BBA como modalidade de gerenciamento de resultados por meio de evidências empíricas de turnover de executivos. Para a detecção de gerenciamento de resultado será empregado o modelo de Dechow et al. (2012), em razão da sua robustez e potencialidade de capturar o BBA. Para tanto, o estudo foca-se em períodos que ocorreram o turnover de executivos e em períodos posteriores ao evento, em um cenário entre os anos 2009 e 2014.

As implicações práticas estão relacionadas com a verificação do emprego da discricionariedade em momentos de situação controversa por empresas de mercado aberto. Uma forma de acalmar o mercado com um novo executivo, demonstrar aparente melhora dos resultados da empresa em período posterior e resiliência organizacional frente à situação controversa ocorrida.

\section{BIG BATH ACCOUNTING (BBA) NO TURNOVER DE EXECUTIVOS}

Empresas que gerenciam resultados tendem a apresentar características de BBA para reconhecer todos os seus accruals em um único período, com o objetivo de no futuro aumentar os resultados. Esse fato ocorre porque os gestores possuem o poder de determinar quando um evento será evidenciado na contabilidade e quais transações afetarão os resultados 
divulgados, como, por exemplo, a apropriação de determinada despesa, receita e alienação de ativos (Watts \& Zimmerman, 1990).

O gerenciamento de resultados é oriundo do comportamento de oportunismo do gestor. Ocorre quando os administradores usam julgamento sobre a informação financeira e sobre as atividades operacionais para alterar informações financeiras, iludir investidores a respeito do desempenho econômico da companhia, ou, ainda, para influenciar resultados contratuais que dependem de números contábeis reportados (Healy \& Wahlen, 1999). Já Martinez (2001) descreve que o gerenciamento de resultados pode ser visto como um modo de atingir uma determinada meta, tanto para aumentar quanto para diminuir o resultado do período (Target Earnings); uma forma de suavizar os resultados, com a redução de variações de resultados (Income Smoothing), ou ainda uma maneira de piorar os resultados atuais em prol de lucros futuros (Big Bath Accounting (BBA)).

Sunder (2014) esclarece que o objetivo do BBA é o de piorar o resultado atual a fim de melhorar o resultado futuro. Nessa prática, a preocupação do gestor visa atender às normas e princípios contábeis, pautados, essencialmente, no conservadorismo. Esse líder, para se manter no poder, conforme Maquiavel (2014) "deve-se cometer todas as crueldades de uma só vez, para não ter que voltar a elas todos os dias [...] Os benefícios devem ser oferecidos gradualmente, para que possam ser melhor apreciados" (p. 60).

Os executivos podem eleger o momento do reconhecimento de accruals discricionários, com o propósito de provocar o big bath, argumentando que o ano foi ruim para o setor ou que algum problema isolado ocorreu na empresa, para, assim, facilmente explicá-lo para agentes externos como uma consequência de fatores fora do controle da gestão, de forma que se pode culpar a gestão anterior e justificar a instalação de uma nova equipe gerencial (Bornemannet al., 2015). Dechow et al. (2012) argumentam que a administração pode gerenciar lucros por vários trimestres anteriores a um determinado evento, e promover um big bath no quarto trimestre após o evento. Já os resultados de Dal Magro et al. (2018) mostram indícios de que, nos primeiros anos de mandato, os executivos são mais propensos a usar práticas oportunistas que envolvem discricionariedade, enquanto para executivos que mantêm longo mandato na organização, ocorrem a manutenção da qualidade da informação contábil e a redução por conflitos de agência.

Durante o mandato de um executivo existe uma relação negativa com o gerenciamento de resultado quando este é observado em um período de curto prazo de gestão - a realização do gerenciamento de resultado aumentará a sua probabilidade de demissão (Hazarika et al., 2012). Já em contextos nos quais existam maior probabilidade de ocorrer rotatividade de executivos forçada em anos subsequentes, ocorre uma relação positiva com o gerenciamento de resultados, sendo esta mais forte se os conselhos da companhia agirem de forma proativa para disciplinar os gestores a gerenciarem os lucros de forma agressiva, mesmo que as manipulações apresentem consequências externas representativas (Hazarika et al., 2012). De 
acordo com os argumentos destacados, apresenta-se a primeira hipótese: $H_{1}$ - A mudança do executivo provoca o BBA em razão da variação nos accruals discricionários.

A cúpula administrativa de uma organização é que ajuda a formar as estratégias que serão seguidas por todos, ao promulgar metas e objetivos, estabelecer uma cultura para a empresa, apresentar a responsabilidade perante a empresa, alterar o desempenho organizacional e atribuir responsabilidades para o próprio gestor de topo (Messersmith, Lee, Guthrie, \& Ji, 2014).

Para Sunder (2014), o gestor possui responsabilidade e poderes para executar e avaliar a mensuração de lucros, e, nesse processo, pode haver a oportunidade de adotar determinadas práticas com o propósito de gerenciá-los. Dessa forma, os alvos são as práticas contábeis discricionárias, pelas quais seja possível interferir na fidedignidade de sua mensuração, a fim da maximização de seu bem-estar pessoal. Esse argumento é válido porque são eles que decidem sobre diversos aspectos na empresa, incluindo a contabilidade, o controle e, sobretudo, questões que demandam julgamento subjetivo, o que pode enviesar as decisões. Messersmith et al. (2014) também discutiram sobre a importância da equipe de gestão de topo na definição dos objetivos estratégicos para a empresa e no estabelecimento de uma cultura organizacional sólida e que sirva de interface entre a organização e seu ambiente externo.

Para Fiordelisi e Ricci (2014), a cultura corporativa pode influenciar a mudança do executivo e, da mesma forma, consegue moderar o desempenho da companhia, principalmente se a cultura corporativa está orientada para a eficácia organizacional, para respostas rápidas para o mercado e com foco na geração de oportunidades futuras. Assim, existirá uma tendência para diminuir o desempenho da empresa em momentos de um turnover de executivos, quando o intuito é realizar melhorias internas da eficiência, controle e implementação de melhores processos. Tendências fortes também estão ligadas às práticas da governança corporativa. Volpin (2002) expõe que a governança corporativa é considerada um mecanismo para evitar a rotatividade do executivo, principalmente se a governança corporativa da organização for forte. Coffee (1999) esclarece que o sistema de governança eficiente pune os executivos das empresas que possuem desempenho baixo ou fluxos de caixa pouco significativos.

A cultura corporativa também pode impactar o desempenho da empresa em momentos de rotatividade de executivos, em especial se forem realizados treinamentos de funcionários internos para assumirem altos cargos (insiders). Esse procedimento afeta negativamente a probabilidade de contratação de um funcionário externo (outsider), evitando gastos expressivos com o monitoramento posterior à contratação (Fiordelisi \& Ricci, 2014).

Por meio desse argumento, a empresa poderá realizar um trabalho de treinamento e capacitação de pessoal (insiders) para futura ocupação de cargos específicos e evitar 
situações em que o futuro gestor possa realizar o gerenciamento de resultados. Assim, a segunda hipótese prediz: $\mathrm{H}_{2}-\mathrm{O}$ novo executivo outsider acaba por apresentar mais gastos discricionários do que o executivo insider.

\section{METODOLOGIA}

Para identificar em que medida a mudança de executivos em empresas listadas na B3 está associada com o BBA, foi realizada uma busca na base de dados $B$ loomberg ${ }^{\circledR}$, obtendose informações de 424 empresas, as quais foram consideradas como população da pesquisa. A base de dados proporcionou o nome dos executivos por empresa, a idade, as datas de início e de fim de atuação na organização, bem como informações de cargos anteriores à ocupação de executivo da companhia. Com as informações coletadas, foi definida a primeira amostra deste estudo, composta de 342 empresas, já que nem todas as empresas possuíam todos os dados necessários para serem incluídas no estudo.

O segundo procedimento realizado foi a separação dos dados para a identificação do BBA, utilizando-se para isso as informações anuais das empresas da amostra. Para tanto, identificou-se a métrica adequada para tal modalidade de gerenciamento de resultados. Dechow et al. (2012) propõem a incorporação de reversões dos accruals nos modelos de gerenciamento de resultado com o objetivo de melhorar a especificação e o poder de explicação. A melhoria aqui está nas variáveis dummy usadas para refletir a reversão nos anos subsequentes. De acordo com os autores, os problemas da omissão de variáveis nos modelos GR podem ser contornados pela adição de reversões. O modelo de Dechow et al. (2012) incorpora períodos durante os quais haja um determinante hipotético de gerenciamento de resultados, sendo, neste estudo, o turnover de executivos.

Para o modelo de definição dos accruals não discricionários de Dechow et al. (2012), é necessária a utilização de uma métrica já existente. Entre as sugestões propostas pelos autores, optou-se pelo modelo de Jones modificado (Dechow, Sloan, \& Sweeney, 1995). Dechow et al. (2012) destacam que a seleção do modelo para a definição dos accruals não discricionários tem pouco impacto sobre o poder do teste, mas que a sua escolha deverá considerar características econômicas que possam ser correlacionadas com a hipótese de gerenciamento de resultados. O modelo de Jones modificado (Dechow et al., 1995) foi escolhido por estar relacionado ao crescimento da empresa e empregar uma variável de contas a receber que apresenta a possibilidade de ser tratada pela empresa (Dechow et al., 1995). O modelo de Jones modificado pode ser representado pela seguinte equação: 


$$
T A_{i, t}=\beta_{0}\left(\frac{1}{A_{i, t-1}}\right)+\beta_{1}\left(\frac{\Delta R_{i, t}-\Delta C R_{i, t}}{A_{i, t-1}}\right)+\beta_{2}\left(\frac{P P E_{i, t}}{A_{i, t-1}}\right)+\varepsilon_{i, t}
$$

Em que:

$T A_{i, t} T A_{i, t}=$ accrual totais da empresa da empresa i no período $\mathrm{t} ;$

$A_{i, t-1} A_{i, t-1}=$ ativo total da empresa i no período anterior;

$\Delta R_{i, t} \Delta R_{i, t}=$ variação das receitas líquidas da empresa i no período $\mathrm{t}$;

$\Delta C R_{i, t} \Delta C R_{i, t}=$ variação das contas a receber da empresa i no período t;

$P P E_{i, t} P P E_{i, t}=$ representa $\circ$ total de propriedade, plantas e equipamento da empresa i no período t;

$$
\beta_{0}, \beta_{1}, \beta_{2} \beta_{0}, \beta_{1}, \beta_{2}=\text { os coeficientes a serem estimados. }
$$

De acordo com os procedimentos realizados por Dechow et al. (2012), também adotados para o presente estudo, foram excluídas da amostra as empresas financeiras, porque o capital de giro destas é menos significativo (redução de 56 empresas na amostra que compunham dados para o cálculo de gerenciamento de resultados e turnover de executivos). Em seguida, os autores também recomendam uma análise de matrizes de covariância por heterocedasticidade, para a identificação de comportamento heterocedástico para todas as observações.

Após todos os procedimentos para a detecção do gerenciamento de resultado $e$ a exclusão de empresas financeiras, o alinhamento das informações sobre o turnover de executivos e a exclusão de outliers, chegou-se ao número de 254 empresas como amostra final. O período a ser pesquisado compreendeu os anos 2009 (ano em que entraram em vigor as normas internacionais de contabilidade no Brasil) a 2014.

No modelo de Dechow et al. (2012) há a exploração de prazos em que haja determinantes hipotéticos de gerenciamento de resultados e evidências para que se faça a reversão para períodos posteriores ao evento, e prova com testes que a incorporação de reversões para períodos posteriores aumenta o poder explicativo do modelo em $40 \%$ e mitiga variáveis omitidas correlacionadas pela não especificação de informações da empresa.

Para efeitos da realização do modelo, são considerados três cenários sobre o momento da reversão: o ano da ocorrência, o ano seguinte e dois anos após o turnover ocorrido. Os recortes consideram a abordagem realizada por Dechow et al. (2012). Para melhor compreensão, apresenta-se a equação a ser utilizada na pesquisa. 


$$
W C_{-} A C C_{i, t}=a+\beta_{1}\left(\frac{\Delta R_{i, t}-\Delta C R_{i, t}}{A_{i, t-1}}\right)+\beta_{2}\left(\frac{P P E_{i, t}}{A_{i, t}-1}\right)+\beta_{3} P A R T_{i, t}+\beta_{4} P A R T 1_{i, t}+\beta_{5} P A R T 2_{i, t}+\varepsilon_{i, t}
$$

Em que:

$W C \_A C C_{i t}=W C \_A C C_{i t}=$ Acréscimo de capital de giro não monetário da empresa i no período t;

$\Delta R_{i, t} \Delta R_{i, t}=$ variação das receitas líquidas da empresa i no período t;

$\Delta C R_{i, t} \Delta C R_{i, t}=$ variação das contas a receber da empresa i no período $\mathrm{t} ;$

$P P E_{i, t} P P E_{i, t}=$ representa o total de propriedade, plantas e equipamento da empresa i no período t;

$A_{i, t-1} A_{i, t-1}=$ ativo total da empresa i no período anterior;

$P A R T_{i t}=$ variável dummy definida como 1 em período em que um determinante hipotético de gerenciamento de resultado está presente da empresa i no período t, e 0 caso contrário;

$P A R T 1_{i t}=$ variável dummy definida como 1 para o primeiro ano após o gerenciamento de resultado estar presente da empresa i no período t, e 0 caso contrário;

$P A R T 2_{i t}=P A R T 2_{i t}=$ variável dummy definida como 1 no segundo ano após o gerenciamento de resultado estar presente na empresa i no período t, e 0 caso contrário;

a, $\beta_{1}, \beta_{2}, \beta_{3}, \beta_{4}, \beta_{5} a, \beta_{1}, \beta_{2}, \beta_{3}, \beta_{4}, \beta_{5^{\prime}}=$ os coeficientes a serem estimados.

Para as variáveis $P A R T_{i t} P A R T_{i t}$, utiliza-se a variável dummy igual a 1 para o período que ocorreu turnover de executivos e 0 caso contrário; $P A R T 1_{i t}, P A R T 1_{i t}$, igual a 1 para identificar se um ano após o fato ocorreu outro turnover de executivo e 0 caso contrário; e PART2 $2_{i t} P A R T 2_{i t}$ igual a 1 se no segundo ano após ocorreu o turnover do executivo e 0 caso contrário. Esse procedimento está alinhado com o pressuposto de que a mudança de executivos está associada com o BBA, especialmente no caso da contratação de executivos outsiders, acompanhando os estudos de Hazarika et al. (2012), Bornemann et al. (2015) e Sunder (2014). A variável dependente $W C_{-} A C C_{i t}=W C_{-} A C C_{i t}=$ Acréscimo de capital de giro não monetário é medida de acordo com a seguinte fórmula:

$$
W C \_A C C_{i, t}=\left(\Delta C A_{i, t}-\Delta C L_{i, t}-\Delta \operatorname{Cash}_{i, t}+\Delta S T D_{i, t}\right) / A_{i, t-1}
$$

Em que:

$\Delta C A_{i, t} \Delta C A_{i, t}=$ variação do ativo circulante da empresa i no período t;

$\Delta C L_{i, t} \Delta C L_{i, t}=$ variação do passivo circulante da empresa i no período $\mathrm{t}$; 
$\Delta \operatorname{Cash}_{i, t} \Delta \operatorname{Cash}_{i, t}=$ variação de caixa e equivalentes de caixa da empresa i no período t; $\Delta S T D_{i, t} \Delta S T D_{i, t}=$ variação da dívida de curto prazo da empresa i no período t;

$A_{i, t-1} A_{i, t-1}=$ ativo total da empresa i no período anterior.

Como variável de controle, utilizou-se apenas o tamanho da empresa (logaritmo natural do Ativo ( $(n A A)$ ). Para o tamanho da empresa, espera-se que, quanto maior a empresa, menor o gerenciamento de resultados (Dechow et al., 2012). Segundo Weigrandt, Kieso, e Kimmel (2005), o log do ativo pode ser utilizado para identificar o porte de uma organização em razão dos seus ativos. Esse procedimento para representar o porte (tamanho) é utilizado em diversos estudos, como os de Nardi e Nakao (2009), Rezende e Nakao (2012), Mendes (2015) e Linhares, Costa, e Beiruth (2018), para citar algumas das pesquisas realizadas no Brasil. Dechow et al. (2012) destacam diversas características econômicas encontradas em estudos sobre gerenciamento de resultados, entre as quais, o tamanho (porte) da empresa é identificado como uma variável extremamente importante.

Para a primeira hipótese deste estudo: $\mathrm{Hl}$ - A mudança do executivo provoca Big Bath Accounting em razão da variação de accruals discricionários no período.

Espera-se que a hipótese apresentada seja aceita, em consonância com evidências empíricas, expostas por Hazarika et al. (2012), Fiordelisi e Ricci (2014) e Bornemann et al. (2015), sugerindo que as despesas discricionárias são mais elevadas no ano de entrada do executivo, ao procurar sinalizar sua capacidade gerencial e a fim de garantir a sua nova posição, principalmente durante a fase inicial de sua administração. No que se refere ao gestor que assumirá o cargo, apresenta-se a hipótese a seguir:

$\mathrm{H} 2$ - O novo executivo outsider utiliza mais gastos discricionários do que o executivo insider.

Espera que essa hipótese também seja aceita, de acordo com Bornemann et al. (2015), cujos resultados apontam que os executivos outsiders (CEO's que assumem não eram do quadro da organização) aumentam ainda mais as despesas discricionárias durante o seu primeiro ano no cargo, ao contrário do executivo insider (novo CEO que já pertencia ao quadro da organização), que pode ter sido treinado para o cargo. Ao gestor insider é atribuído o valor dummy 0), ao passo que ao CEO outsider é atribuído o valor dummy 1.

Vale ressaltar que, para a análise dos dados, utilizou-se o software Statistical Package for Social Sciences (SPSS) ${ }^{\circledR}$, versão 22, com o objetivo de efetuar análises descritivas, verificação de outliers, testes de normalidade e verificação de homogeneidade das variâncias, além do software Gretl, versão 1.10.1, para a análise de dados em painel. 


\section{ANÁLISE DOS DADOS}

Com os achados, é possível realizar uma análise descritiva sobre o turnouver dos executivos da amostra. Entre as 254 empresas da amostra final, no período de 2009 a 2014, identificou-se a existência de 324 executivos que passaram pelas empresas como CEOs ou que ainda fazem parte dessas entidades. Para identificar as especificidades dos executivos, foi realizado um levantamento da idade, período na empresa em anos, tipos de CEOs contratados e quantidade de turnover total e por ano, conforme detalhado na Tabela 1:

Tabela 1

Informações qualitativas sobre o turnover de executivos

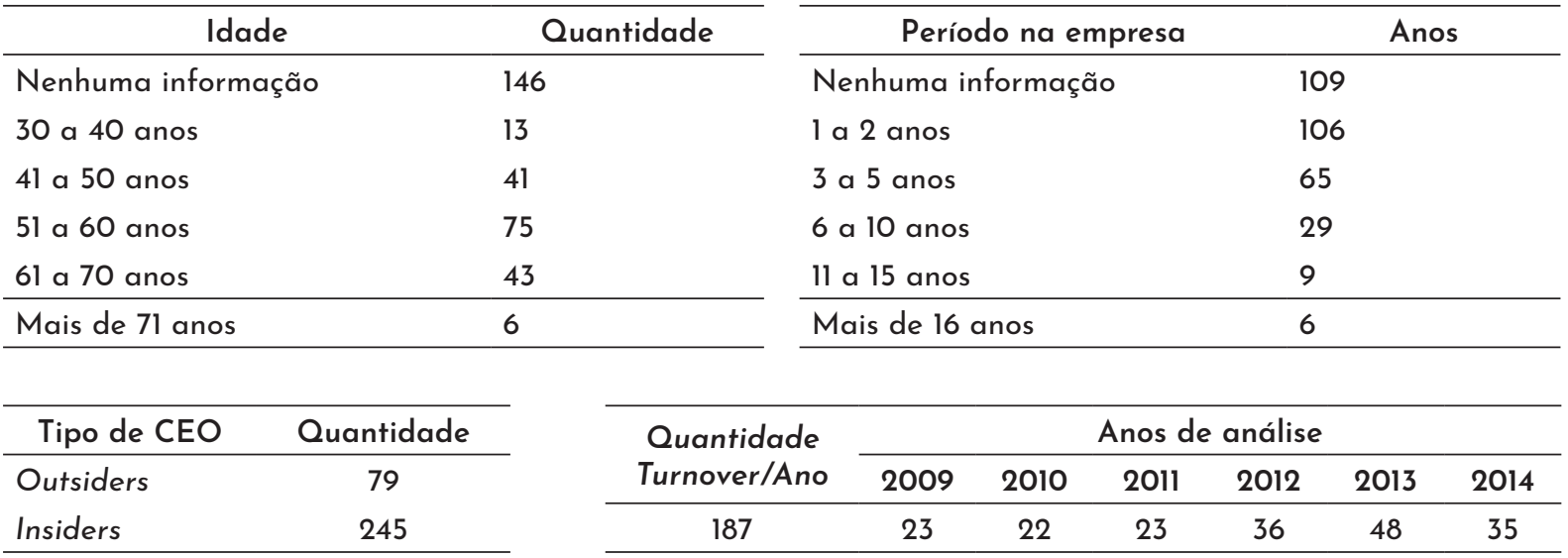

Com relação à idade média dos 324 executivos, a maioria apresenta idade maior que 50 anos, com destaque aos seis executivos com mais de 70 anos. Esses dados informam que, para esse cargo, as pessoas tendem a ser mais velhas. Todavia, percebe-se uma grande rotatividade de executivos nas empresas de mercado aberto brasileiras, por haver uma representatividade na permanência de um a dois anos, e uma baixa na quantidade de pessoas que ocupam o cargo de CEO há mais de dez anos. Vale ressaltar que essa grande rotatividade de executivos dificulta o estabelecimento de uma cultura organizacional sólida (Messersmith et al., 2014) e acaba por proporcionar altos custos transacionais com as mudanças de aspectos estratégicos na companhia, treinamentos, custos trabalhistas rescisórios e adaptação de novos colaboradores para o cargo, assim como um fator motivador para a utilização de despesas sobrecarregadas (Sunder, 2014).

Percebe-se que a maioria dos executivos é nomeada internamente para a função de CEO, ou seja, o executivo já exercia cargos e funções na empresa anteriores à nomeação de executivo (insider). Esse fato comprova a necessidade de uma pessoa de alta confiança, com experiência e bom relacionamento interno na organização, o que contribui para a formação de uma cultura corporativa, que já se prepara para casos de rotatividade, por meio da realização de treinamento com colaboradores internos para assumir altos cargos em 
períodos posteriores (Fiordelisi \& Ricci, 2014). Por fim, observa-se a quantidade de turnovers que ocorreu ao longo dos seis anos abrangida pela pesquisa. Nas 324 empresas abrangidas na amostra, ocorreram 187 trocas de gestores no período entre 2009 e 2014. Entre essas trocas, o ano que apresentou a maior representatividade foi 2013, com 48 trocas, mas que pode ser reflexo de um aumento que já vinha sendo notado no ano 2012.

Com a coleta de dados das companhias listadas para o período de 2009 a 2014 (informações anuais) e a exclusão das empresas do setor financeiro e daquelas que não apresentaram os dados necessários para a realização das análises, verificou-se, na sequência, a existência de dados extremos (outliers) e as verificações da normalidade dos dados e da homogeneidade das variâncias. Para a verificação dos outliers, foi realizada uma tabulação dos dados e cálculo das variáveis necessárias para a viabilização dos modelos, com a realização de correção deles pela substituição destes, de acordo com o valor da soma da média mais dois desvios padrão, conforme indicação de Field (2009).

Com relação à normalidade, após o ajuste dos outliers as variáveis se mostraram comportadas. A verificação da normalidade segue o parâmetro dos valores entre $-1 e+1$ para assimetria e entre -3 e +3 para curtose, segundo parâmetros indicados por Hair, Babin, Money, e Samouel (2005). A análise gráfica para a constatação da não violação da suposição de normalidade dos dados teve em vista os preceitos do teste de KolmogorovSmirnov, normalmente utilizado para testar a normalidade dos dados em grandes amostras, o que não indicou a normalidade, o que já era esperado, pois segundo Hair, Black, e Babin (2009), esses testes são muito sensíveis em amostras grandes.

A homogeneidade das variâncias foi realizada pelo Teste de Levene, no entanto, da mesma forma que no caso da normalidade, grandes amostras podem comprometer os resultados (FIELD, 2009). Assim, utilizou-se a metodologia apontada por Field (2009), em que o resultado da razão entre o grupo com maior variância e o grupo com a menor variância "for menor que 2, é seguro afirmar que há homogeneidade das variâncias" (p. 117).

Na sequência, os dados foram importados para o software de análise estatística Gretl para a realização de regressão de dados em painel. Na análise por meio desse software, foram desconsideradas as linhas que continham alguma informação faltante, assim como o modelo pelo MQO agrupado, de acordo com 1.407 observações e 254 unidades de corte transversal, a partir da Equação 2. Os resultados são apresentados na Tabela 2, que já considera a variável de controle tamanho da empresa e as variáveis dummy representativas da ocorrência de turnover, conforme especificado anteriormente. 
Tabela 2

Estimativa inicial do modelo (MQO agrupado)

\begin{tabular}{ccccc}
\hline Variáveis & Coeficientes & Erro padrão & Razão $\mathbf{~}$ & p-value \\
\hline Constante & $-0,0186$ & 0,01736 & $-1,069$ & 0,285 \\
\hline$\left(\Delta \mathbf{R}_{i, t}-\Delta \mathbf{C R}_{i, t}\right) / \mathbf{A}_{i,-1-1}$ & 0,0117 & 0,04687 & 0,2503 & 0,802 \\
\hline $\mathbf{P P E}_{i, t} / \mathbf{A}_{i,+-1}$ & $-0,0135$ & 0,01212 & $-1,111$ & 0,267 \\
\hline LogA & 0,0042 & 0,00204 & 2,053 & $0,040^{*}$ \\
\hline Part & $-0,0137$ & 0,01077 & $-1,272$ & 0,204 \\
\hline Part1 & $-0,0014$ & 0,01137 & $-0,1267$ & 0,899 \\
\hline Part2 & $-0,0016$ & 0,01635 & $-0,0963$ & 0,923
\end{tabular}

Nota. ${ }^{*}$ Significantes ao nível de 0,05

Os resultados foram submetidos aos testes Chow, Breusch-Pagan e de Hausman para identificação da necessidade de utilização de modelo de efeitos fixos, aleatórios ou polled, conforme sugerido por Fávero (2009). A estatística de teste de Breusch-Pagan ( $p$-value = $0,797)$ indica que o modelo adequado para as análises é o MQO agrupado, permanecendo os resultados apresentados na Tabela 1 . Os resultados apresentados não indicam qualquer interferência da ocorrência de turnover no gerenciamento de resultado, a partir da Equação 1, proposta por Dechow et al. (2012).

Observou-se que apenas uma das variáveis do modelo se mostrou estatisticamente significante, sendo essa variável representada pelo total dos ativos, que representa o porte da empresa. Esse resultado indica que apenas o porte exerce papel relevante na explicação das variações dos acréscimos do capital de giro. De modo geral, de acordo com o modelo, não há uma identificação que a mudança do executivo da empresa esteja associada com acréscimos do capital de giro. Apesar da não significância das variáveis relacionadas ao turnover, o sinal apresentado por elas pode dar alguns indícios interessantes.

A primeira dummy $P A R T_{i t} P A R T_{i t}$, que representa o período (ano) em que ocorreu turnover, indica um sinal negativo em seu coeficiente, o que representa uma relação inversamente proporcional com acréscimo de capital de giro não monetário, ou seja, quando há turnover em uma empresa, ocorre uma diminuição do capital de giro. Esse fato sugere indícios de ocorrência de um BBA, no período, conforme também apontam Hazarika et al. (2012), Fiordelisi e Ricci (2014) e Bornemann et al. (2015).

Tal característica também é evidenciada um ano após a ocorrência do turnover na empresa, o que demonstra a utilização de acúmulos discricionários com um ano após a ocorrência da mudança do executivo, mesmo que o coeficiente encontrado seja menos quando comparado com o ano em evidencia. Esse fato é explicado pelo modelo de Dechow et al. (2012).

Quando analisado o coeficiente de correção do segundo ano após a ocorrência de mudança do CEO, a relação começa a se estabilizar com uma variação positiva entre esse turnover e acréscimo de capital de giro não monetário. Entre comportamento de uma 
variação negativa no ano da ocorrência do turnover, com reflexos no ano seguinte e uma variação positiva no segundo ano, comprova-se a existência do BBA (Bornemann et al., 2015). Todavia, essa constatação não pode ser aprovada por não haver representatividade estatística nos resultados.

Outro aspecto de destaque, apesar da não significância estatística do modelo, é a relação negativa que ocorre entre o tamanho da empresa e os acréscimos do capital de giro, fato descrito no estudo de Dechow et al. (2012), de que, quanto maior a empresa, menor os indícios de gerenciamento de resultados, o que demonstra uma contribuição desse estudo, de acordo com a amostra pesquisada.

Para uma maior robustez na análise dos dados, optou-se por analisar as mesmas informações e dados por uma diferente perspectiva, de acordo com especificações descritas na Equação 1. Para tal cálculo, a intenção é identificar se, no período em que houve rotatividade do executivo, ocorreu gerenciamento de resultados; para isso, empregou-se o Modelo de Jones modificado como variável dependente e os períodos em que houve turnover de executivos, com variáveis independentes.

$$
T A_{i, t}=\beta_{0}\left(\frac{1}{A_{i, t-1}}\right)+\beta_{1}\left(\frac{\Delta R_{i, t}-\Delta C R_{i, t}}{A_{i, t-1}}\right)+\beta_{2}\left(\frac{P P E_{i, t}}{A_{i, t}-1}\right)+\beta_{3} P A R T_{i, t}+\beta_{4} P A R T 1_{i, t}+\beta_{5} P A R T 2_{i, t}+\varepsilon_{i, t}
$$

Em que:

$T A_{i, t} T A_{i, t}=$ accruals totais da empresa da empresa i no período t;

$A_{i, t-1} A_{i, t-1}=$ ativo total da empresa i no período anterior;

$\Delta R_{i, t} \Delta R_{i, t}=$ variação das receitas líquidas da empresa i no período $\mathrm{t}$;

$\Delta C R_{i, t} \Delta C R_{i, t}=$ variação do contas a receber da empresa i no período t;

$P P E_{i, t} P P E_{i, t}=$ representa o total de propriedade, plantas e equipamento da empresa i no período t;

$P A R T_{i t}=P A R T_{i t}=$ variável dummy definida como 1 em período em que um determinante hipotético de gerenciamento de resultado está presente da empresa $\mathrm{i}$ no período t, e 0 caso contrário; $P A R T 1_{i t}=P A R T 1_{i t}=$ variável dummy definida como 1 para o primeiro ano após o gerenciamento de resultado estar presente da empresa i no período t, e 0 caso contrário;

$P A R T 2_{i t}=P A R T 2_{i t}=$ variável dummy definida como 1 no segundo ano após o gerenciamento de resultado estar presente na empresa i no período t, e $O$ caso contrário;

$\beta_{0}, \beta_{1}, \beta_{2}, \beta_{3}, \beta_{4}, \beta_{5}=$ os coeficientes a serem estimados. Os resultados obtidos a partir desse modelo são apresentados na Tabela 3: 
Tabela 3

Estimativa do modelo de Jones modificado

\begin{tabular}{|c|c|c|c|c|}
\hline Variáveis & Coeficientes & Erro padrão & Razão † & p-value \\
\hline $1 / A_{i, t-1}$ & 0,0741 & 0,03705 & 2,001 & $0,046^{* *}$ \\
\hline$\left(\Delta \mathrm{R}_{\mathrm{i}, \mathrm{t}}-\Delta \mathrm{CR}_{\mathrm{i}, \mathrm{t}}\right) / \mathrm{A}_{\mathrm{i}, \mathrm{t}-\mathrm{I}}$ & 0,1833 & 0,08414 & 2,179 & $0,030^{* *}$ \\
\hline $\operatorname{PPE}_{i, t} / A_{i, t-1}$ & $-0,3562$ & 0,04171 & $-8,541$ & $0,000^{* * *}$ \\
\hline $\log A$ & 0,0626 & 0,03704 & 1,691 & $0,091^{*}$ \\
\hline Part & 0,0097 & 0,02100 & 0,4602 & 0,645 \\
\hline Partl & 0,0286 & 0,02586 & 1,107 & 0,268 \\
\hline Part2 & 0,0047 & 0,03989 & 0,1189 & 0,905 \\
\hline
\end{tabular}

Nota. ${ }^{*}$ Significantes ao nível de 0,10; ${ }^{* *}$ Significantes ao nível de 0,05; ${ }^{* * *}$ Significantes ao nível de 0,01.

Diferente do modelo anterior, na Tabela 3 as variáveis principais foram estatisticamente significantes, inclusive a variável de controle (tamanho da empresa), o que indica uma maior relevância na explicação das variações dos accruals totais. No entanto, também nesse caso - turnover não se mostrou estatisticamente significante. Para tal resultado, não se tem evidência de gerenciamento de resultados, de acordo com a modalidade de alisamento ou suavização de resultados em períodos em que ocorre uma mudança de executivos na empresa, fato indicado nos estudos de Eckel (1981) e Leuz, Nanda, e Wysocki (2003).

Com relação ao tamanho da empresa e os accruals totais, para o cálculo realizado a variação é positiva e significante, indicando que a variação dos accruals totais aumenta à medida que aumenta o tamanho.

A partir dos resultados apresentados, com o emprego de dois modelos distintos, não foi possível constatar que a mudança do executivo provoca a ocorrência de Big Bath Accounting em razão da variação de accruals discricionários no período, o que não possibilita confirmar ou refutar a hipótese proposta $(\mathrm{H} 1)$. A partir dessa evidência empírica, não foi possível comparar com os achados descritos por Hazarika et al. (2012), Fiordelisi e Ricci (2014) e Bornemann et al. (2015).

Para buscar evidências que expliquem que a origem do CEO influencia maiores gastos discricionários, houve a separação dos executivos insider, quando caracterizado por um colaborador treinado para o cargo ou que já exercia outras funções internas antes da mudança, e/ou outsider, quando contratado e advindo de outra organização especificamente para exercer essa função. Dessa forma, a hipótese $\mathrm{H} 2$, baseada nas evidências de Bornemann et al. (2015), indica que executivos outsider apresentam maiores gastos discricionários nos primeiros anos no cargo de Chefe-executivo.

Com o emprego do último modelo (Equação 4), também foi possível verificar a não existência de diferença entre executivos insiders e outsiders para a utilização de mais gastos discricionários. Para comprovar tal informação, foi realizada a análise para as empresas que contrataram executivos outsiders em momentos em que ocorreram turnover na empresa, conforme apresentado na Tabela 4: 
Tabela 4

Estimativa do modelo de Jones modificado em empresas que contrataram outsiders

\begin{tabular}{|c|c|c|c|c|}
\hline Variáveis & Coeficientes & Erro padrão & Razão t & p-value \\
\hline $1 / A_{i, t-1}$ & 0,1900 & 0,08574 & 2,216 & $0,030^{* *}$ \\
\hline$\left(\Delta \mathbf{R}_{i, t}-\Delta \mathbf{C R}_{i, t}\right) / \mathbf{A}_{i, t-1}$ & $-0,3996$ & 0,28937 & $-1,381$ & 0,171 \\
\hline $\mathrm{PPE}_{\mathrm{i}, \mathrm{t}} / \mathrm{A}_{\mathrm{i}, \mathrm{t}-\mathrm{l}}$ & $-0,3350$ & 0,08227 & $-4,072$ & $0,000^{* * *}$ \\
\hline $\log A$ & 0,1704 & 0,08619 & 1,977 & $0,052^{*}$ \\
\hline Turnover (outsider) & 0,0382 & 0,06980 & 0,548 & 0,585 \\
\hline
\end{tabular}

Nota. ${ }^{*}$ Significantes ao nível de 0,$10 ;{ }^{* *}$ Significantes ao nível de 0,$05 ;{ }^{* * *}$ Significantes ao nível de 0,01 .

Um fato que pode comprovar a não apresentação de significância é a grande redução da amostra para esse cálculo, composta por somente 86 observações. Um aspecto interessante é o sinal positivo nos coeficientes relativo à variável turnover, quando o executivo é um outsider, o que vai de encontro aos resultados de Bornemann et al. (2015), que apresentou uma relação negativa entre turnover de outsiders e gerenciamento de resultados. Assim, também não é possível afirmar que com o novo executivo outsider existe a ocorrência de mais gastos discricionários do que com o executivo insider, o que nos proporciona subsídios para a refutação da hipótese proposta.

Além dos resultados apresentados, o modelo de Jones modificado também apresentou resultados semelhantes, mesmo com a possibilidade de identificar $\circ \varepsilon_{i, t,}$ que representa os accruals discricionários. A partir da identificação dos achados, é possível dizer que independe a origem do executivo, ou seja, essa proxy não indica a ocorrência de maiores gastos discricionários.

\section{CONCLUSÕES}

O estudo tratou de identificar em que medida a mudança de executivos em empresas listadas na B3 está associada com o BBA, partindo-se da hipótese de que a modalidade de gerenciamento de resultados $B B A$ resulta em provisionamentos intencionais de despesas em determinados períodos, com o intuito de reduzir o lucro em um ano quando já se sabe que a empresa está em um período ruim.

Com um montante de 1.408 observações e a partir de uma amostra final de 254 empresas listadas na B3, em um período de seis anos (entre 2009 e 2014), foram identificadas, na base de dados Bloomberg ${ }^{\circledR}$, as informações necessárias para a identificação dos eventos de turnover de executivos, assim como os dados necessários para a identificação do BBA, calculado de acordo com a métrica de gerenciamento de resultados sugerida por Dechow et al. (2012). 
Quando realizado o teste para identificar efeito do $B B A$ em períodos em que ocorreram turnover de executivos, somente as variáveis de imobilizado e tamanho da empresa apresentaram representatividade em relação a variações dos acréscimos do capital de giro. Entretanto, não há uma identificação de que a mudança do executivo da empresa esteja associada com acréscimos do capital de giro. Dessa forma, os resultados do presente estudo realizado com companhias brasileiras não identificaram resultados semelhantes ao observado em Hazarika et al. (2012), Fiordelisi e Ricci (2014) e Bornemann et al. (2015).

Semelhante teste foi realizado para as empresas que contrataram executivos outsiders, mas nenhuma constatação estatisticamente significante pôde ser confirmada. Desse modo, foi possível estabelecer a conclusão da não existência de medidas significativas que comprovam que a mudança de executivos em empresas listadas na B3 está associada com - $B B A$, sendo os resultados obtidos em companhias brasileiras conflitantes com o observado por Bornemann et al. (2015).

De acordo com os resultados apresentados, argumenta-se como fator limitador desta pesquisa a pequena amostra de empresas analisadas, no qual pode ter enviesado a falta de um $p$-value representativo. Como sugestão para futuras pesquisas, recomenda-se aumentar a amostra de pesquisa, com a inclusão de empresas financeiras ou de empresas estrangeiras que negociam ações em outras bolsas de valores, assim como a utilização do modelo de Dechow et al. (2012) com outras métricas para selecionar os accruals discricionários. Do mesmo modo, ampliar o período de análise para 10 anos, como o período de 2009 a 2019.

Por meio deste trabalho, incentiva-se a quebra de paradigmas no que concerne a essa modalidade de gerenciamento de resultados - BBA. Para isso, recomenda-se a realização de um estudo bibliográfico que empregue um procedimento sistematizado para a gestão de conhecimento e identificação de oportunidades de pesquisa científica, do mesmo modo, a crítica de materiais já publicados sobre esse tema, que não apenas empreguem uma abordagem funcionalista/positivista, mas também abordagens como a interpretativista e a crítica, que possuem a sua contribuição para a consolidação do tema.

\section{REFERÊNCIAS}

Bornemann, S., Kick, T., Pfingsten, A., \& Schertler, A. (2015). Earnings baths by CEOs during turnovers: Empirical evidence from German savings banks. Journal of Banking \& Finance, 53(1), 188-201.

Comitê de Pronunciamentos Contábeis. (2015). CPC OO (RI) - Estrutura Conceitual para Elaboração e Divulgação de Relatório Contábil-Financeiro. Recuperado de http://static. cpc.mediagroup.com.br/Documentos/147_CPCOO_Rl.pdf 
Coffee, J. (1999). The future as history: The prospects for global convergence in corporate governance and its implications. Northwestern University Law Review, 93(1), 641-708.

Linhares, F. S., Costa, F. M., \& Beiruth, A. X. (2018). Gerenciamento de resultados e eficiência de investimentos. Revista Brasileira de Gestão de Negócios, 20(2), 295-310. Recuperado de $\mathrm{http}: / /$ dx.doi.org/10.7819/rbgn.v20i2.3180

Dal Magro, C., Klann, R. C., \& Mondini, V. E. D. (2018). CEOs' extensive term of office inhibits discretionary accruals. RAUSP Management Journal, 53(4), 575-596.

Dechow, P. M., Hutton, A. P., Kim, J. H., \& Sloan, R. G. (2012). Detecting Earnings Management: A New Approach. Journal of Accounting Research, 50(2), 275-334.

Dechow, P. M., Sloan, R. G., \& Sweeney A. P. (1995). Detecting Earnings Management. The Accounting Review, 70(2), 193-225.

Eckel, N. (1981). The income smoothing hypothesis revisited. Abacus, 17(1), 28-40.

Fávero, L. P. (2009). Análise de dados: Modelagem multivariada para tomada de decisões. Rio de Janeiro: Elsevier.

Field, A. (2009). Descobrindo a estatística usando o SPSS (L. Viali Trad.). (2 ${ }^{a}$ ed.). Porto Alegre: Artmed.

Fiordelisi, F. \& Ricci, O. (2014). Corporate culture and CEO turnover. Journal of Corporate Finance, 28(1), 66-82.

Hair, J. F., Jr., Black, W. C., \& Babin, B. J. (2009). Análise multivariada de dados (A. S. Sant'Anna \& A. Chaves Neto Trad.). (6 ${ }^{a}$ ed.). Porto Alegre: Bookman.

Hair, J. F., Jr., Babin, B., Money, A. H., \& Samouel, P. (2005). Fundamentos de métodos de pesquisa em administração (L. Belon Ribeiro Trad.). Porto Alegre: Bookman.

Hazarika, S., Karpoff, J. M., \& Nahata, R. (2012). Internal corporate governance, CEO turnover, and earnings management. Journal of Financial Economics, 104(1), 44-69.

Healy, P. M., \& Wahlen, J. M. (1999). A review of the earnings management literature and its implications for standard setting. Accounting Horizons, 13(4), 365-383. 
Jensen, M. C., \& Meckling, W. (1976). Theory of firm: Managerial behavior, agency costs and ownership structure. Journal of Financial Economics, 3(4), 305-360.

Leuz, C., Nanda, D., \& Wysocki, P. D. (2003). Investor protection and earning management: an international comparison. Journal of Financial Economics, 69(3), 505-527.

Maquiavel, N. (2014). O príncipe. 1469-1527 Tradução (R. Grassi Trad.). (1ª ed.). Rio de Janeiro: BestBolso.

Martinez, A. L. (2001). Gerenciamento dos resultados contábeis: Estudo empírico das companhias abertas brasileiras (Tese de doutorado). Programa Multi-institucional e Inter-regional de Pós-graduação em Ciências Contábeis UnB/UFPB/UFRN, Brasília, DF.

Mendes, C. J. F. (2015). Mensuração a valor justo e gerenciamento de resultados: Uma Análise dos Bancos Comerciais e Múltiplos no Cenário Internacional (Tese de doutorado). Universidade de São Paulo, São Paulo.

Messersmith, J. G., Lee, J., Guthrie, J. P., \& Ji, Y. (2014). Turnover at the Top: Executive Team Departures and Firm Performance. Organization Science, 25(3), 776-793.

Nardi, P. C. C., \& Nakao, S. H. (2009). Gerenciamento de resultados e a relação com o custo da dívida das empresas brasileiras abertas. Revista Contabilidade e Finanças, 20(50), 77-100.

Rezende, G. P., \& Nakao, S. H. (2012). Gerenciamento de resultados e a relação com o lucro tributável das empresas brasileiras de capital aberto. Revista Universo Contábil, 8(1), 6-21.

Sunder, S. (2014). Teoria da contabilidade e do controle. São Paulo: Atlas.

Volpin, P. F. (2002). Governance with poor investor protection: Evidence from top turnover in Italy. Journal of Financial Economics, 64(1), 61-90.

Watts, R. L., \& Zimmerman, J. L. (1990). Positive accounting theory: A ten years perspective. The Accounting Review, 65(1), 131-156.

Weigandt, J. J., Kieso, D. E., \& Kimmel, P. D. (2005). Contabilidade Financeira (E. C. da Silva \& J. L. Paravato Trad. \& J. S. Coutinho \& L. H. B. Machado). (3 ed.). Rio de Janeiro: LTC. 


\section{Como citar este artigo:}

\section{ABNT}

THEISS, Viviane et al. Big Bath Accounting e turnover de executivos em empresas listadas na B3. RACE, Revista de Administração, Contabilidade e Economia, Joaçaba: Ed. Unoesc, v. 18, n. 1, p. 137-156, jan./abr. 2019. Disponível em: https://portalperiodicos.unoesc.edu. br/race. Acesso em: dia/mês/ano.

\section{APA}

Theiss, V., Portulhak, H., Kuhl, M. R., \& Colauto, R. D. (2019). Big Bath Accounting e turnover de executivos em empresas listadas na B3. RACE, Revista de Administração, Contabilidade e Economia, 18(1), 137-156. Recuperado de http://editora.unoesc.edu.br/index.php/ race 